\title{
Insights Into the Controversial Aspects of Adiponectin in Cardiometabolic Disorders
}

Authors

Emilio Antonio Francischetti ${ }^{1}$, Rômulo Sperduto Dezonne ${ }^{2}$, Cláudia Maria Pereira ${ }^{2}$, Cyro José de Moraes Martins ${ }^{1}$, Bruno Miguel Jorge Celoria ${ }^{3}$, Patrícia Aguiar Cardoso de Oliveira², Virgínia Genelhu de Abreu ${ }^{1}$

\author{
Affiliations \\ 1 Laboratory of Clinical and Experimental Pathophysiology, \\ Rio de Janeiro State University, Rio de Janeiro, Brazil \\ 2 Postgraduate Program in Translational Biomedicine, \\ Grande Rio University, Duque de Caxias, Brazil \\ 3 Hospitals Copa Star \& Copa D’Or - Rede D’Or São Luiz, \\ Rio de Janeiro, Brazil
}

\section{Key words}

obesity, insulin resistance, adiponectin resistance, adiponectin paradox, diabetes mellitus

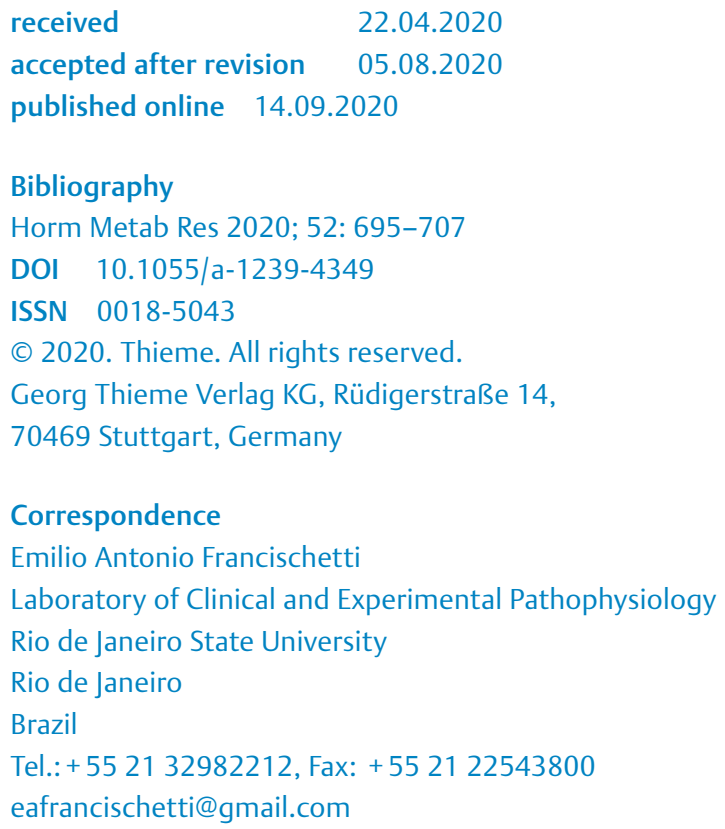

\section{ABSTRACT}

In 2016, the World Health Organization estimated that more than 1.9 billion adults were overweight or obese. This impressive number shows that weight excess is pandemic. Overweight and obesity are closely associated with a high risk of comorbidities, such as insulin resistance and its most important outcomes, including metabolic syndrome, type 2 diabetes mellitus, and cardiovascular disease. Adiponectin has emerged as a salutary adipocytokine, with insulin-sensitizing, anti-inflammatory, and cardiovascular protective properties. However, under metabolically unfavorable conditions, visceral adipose tissue-derived inflammatory cytokines might reduce the transcription of the adiponectin gene and consequently its circulating levels. Low circulating levels of adiponectin are negatively associated with various conditions, such as insulin resistance, type 2 diabetes mellitus, metabolic syndrome, and cardiovascular disease. In contrast, several recent clinical trials and meta-analyses have reported high circulating adiponectin levels positively associated with cardiovascular mortality and allcause mortality. These results are biologically intriguing and counterintuitive, and came to be termed "the adiponectin paradox". Adiponectin paradox is frequently associated with adiponectin resistance, a concept related with the downregulation of adiponectin receptors in insulin-resistant states. We review this contradiction between the apparent role of adiponectin as a health promoter and the recent evidence from Mendelian randomization studies indicating that circulating adiponectin levels are an unexpected predictor of increased morbidity and mortality rates in several clinical conditions. We also critically review the therapeutic perspective of synthetic peptide adiponectin receptors agonist that has been postulated as a promising alternative for the treatment of metabolic syndrome and type 2 diabetes mellitus.

\section{Introduction}

The Global Burden of Disease Study estimated that overweight adults, that is, with a body-mass index (BMI) of $25 \mathrm{~kg} / \mathrm{m}^{2}$ or higher, increased from $28.8 \%$ to $36.9 \%$ in men and from $29.8 \%$ to $38.0 \%$ in women between 1980 and 2013 worldwide [1]. In 2016, more than 1.9 billion adults aged 18 years and older were overweight. Of these, over 650 million were obese [2].
Overweight and obesity are closely associated with a high risk of comorbidities, such as insulin resistance and its most important outcomes, including type 2 diabetes mellitus (T2DM), metabolic syndrome, and cardiovascular diseases [3]. Among the causes of cardiovascular disease, chronic heart failure has been increasing continuously worldwide, mostly as a factor of the increase in life expectancy [4]. Together, these comorbidities increase the mortality rates in weight excess individuals [5]. 
Evidence both from experimental studies and from clinical data suggests that in obesity, the expansion and differentiation of adipocytes cause recruitment of several types of inflammatory cells, leading to increased expression of inflammatory cytokines, with adverse effects such as tumor necrosis factor alpha (TNF- $\alpha$ ), monocyte chemoattractant protein 1 (MCP-1), interleukin 6 (IL-6), lipocalin-2, adipocyte fatty acid-binding protein, and reactive oxygen species (ROS) [6].

Adiponectin has emerged as a major salutary adipocytokine, with insulin-sensitizing [7], anti-inflammatory [8], anti-atherogenic [9], and cardiovascular protective functions [10]. Adiponectin circulates in healthy subjects at very high concentrations, with levels ranging from 3 to $30 \mu \mathrm{g} / \mathrm{ml}$ [11]. However, under metabolically unfavorable conditions, visceral adipose tissue-derived cytokines might reduce the transcription of the adiponectin gene [12] and consequently its circulating levels [13]. Hypoadiponectinemia is negatively associated with various conditions, such as insulin resistance, abdominal obesity and T2DM [14], non-alcoholic fatty liver disease (NAFLD) [15], metabolic syndrome [16], dyslipidemia [17], hypertension [18], and cardiovascular diseases [19].

In contrast, several recent clinical trials and meta-analyses have reported that high circulating adiponectin levels were positively associated with cardiovascular mortality and all-cause mortality in different cohorts of patients and clinical settings [20-23]. High adiponectin levels are also associated with weight loss, low muscle mass, and poor physical functioning among the elderly [24]. As adiponectin has been considered a healthy adipokine, these results are biologically intriguing and counterintuitive, and came to be termed "the adiponectin paradox" [25]. Adiponectin paradox is frequently associated with adiponectin resistance, a concept that was introduced to report the down-regulation of adiponectin receptors in insulin-resistance and obesity [26-27].

Here, we review the contradiction that both basic scientific knowledge and results from earlier observational and prospective clinical studies show adiponectin as a health promoter, whereas recent evidence from Mendelian randomization studies indicates that circulating adiponectin levels are an unexpected predictor of increased morbidity and mortality rates in several clinical conditions.

\section{Adiponectin and Inflammation}

\section{Anti-inflammatory actions of adiponectin}

An extensive body of research suggests that adiponectin acts as an anti-inflammatory mediator in cardiometabolic disorders such as T2DM, hepatic steatosis, and cardiovascular disease. At the cellular level, several processes are involved in the beneficial role of adiponectin in inflammation, including: (i) the inhibition of Toll-like receptor family-induced activation of NFKB in macrophages [28]; (ii) the suppression of TNF- $\alpha$ expression and monocyte chemoattractant protein [29]; (iii) the increase of tissue inhibitor-1 through the anti-inflammatory cytokine IL-10 expression [30]; (iv) the promotion of macrophages polarization and its transformation from a proinflammatory $\mathrm{M} 1$ to an anti-inflammatory $\mathrm{M} 2$ phenotype [30]; (v) the inhibition of transformation of human monocyte-derived macrophages into foam cells, thus protecting against atherogenesis [31]; and (vi) its inhibitor effect on oxidative stress of $\mathrm{db} / \mathrm{db}$ mice aortic endothelial cells by increasing nitric oxide bioavailability through AMPK and cAMP-dependent protein kinase (PKA) [30].

\section{Proinflammatory actions of adiponectin}

Paradoxically and unexpected, adiponectin may exert a deleterious effect related to its capacity in promoting inflammation. However, the proinflammatory action of adiponectin is controversial, particularly in conditions of in vitro assays, under the dependence of experimental conditions such as the source of adiponectin, the molecular weight of adiponectin, and the temporal response of cell (usually macrophages) exposition to inflammatory stimuli [32, 33]. Differently from in vitro studies, the immune system in vivo is permanently submitted to higher circulating levels of adiponectin, which mediate a cell memory effect that induces an anti-inflammatory response [32].

Nonetheless, there is an extensive literature showing both in vitro and in vivo conditions a paradoxically elevated adiponectin concentration in human inflammatory diseases such as chronic inflammatory bowel disease (Crohon's disease) [34], rheumatoid arthritis [35], end stage renal disease [36], hepatic cirrhosis [37] and chronic obstructive pulmonary disease [38]. Although these data may explain the divergent results of in-vitro and in-vivo studies reported in the literature, the precise mechanism of adiponectin proinflammatory actions and in what way adiponectin can also modulate its anti-inflammatory responses are still unknown.

\section{Understanding Adiponectin Resistance}

\section{Adiponectin resistance in obesity and diabetes}

The concept of adiponectin resistance was initially introduced to describe the downregulation of AdipoR1 in insulin-resistant states and obesity [26]. Adiponectin and insulin have numerous metabolic similarities, as both promote glucose uptake and inhibit liver gluconeogenesis ( $>$ Figs. 1,2). In high-fat-diet-induced obesity and insulin-resistant states, insulin-cascade signaling is lessened by excess lipid accumulation in skeletal muscle, impairing mitochondrial bioenergetics and morphology [39]. Extensive published data provide evidence that adiponectin and insulin resistances frequently co-exist, and a vicious circle might be established in certain circumstances. For example, any dysmetabolic condition associated with insulin resistance may result in adiponectin resistance and hyperadiponectinemia, as the insulin/FOXO1 pathway regulates the levels of expression of adiponectin receptors and adiponectin sensitivity [40].

Experimental protocols have extensively confirmed the co-occurrence of adiponectin resistance and insulin resistance. In genetically obese mice, AdipoR1 and AdipoR2 are significantly reduced in several insulin-sensitive tissues such as skeletal muscle, liver, and adipose tissue, compared with non-obese animals. Insulin reduces the expression of AdipoR1 and AdipoR2 via downregulation of FOXO1 phosphorylation, with subsequent attenuation of adiponectin binding and reduction of their metabolic homeostatic actions ( Fig. 3) [26].

Simultaneous disruption of AdipoR1 and AdipoR2 might abolish adiponectin binding and actions, resulting in increased tissue triglyceride content, inflammation, and oxidative stress, thus lead- 


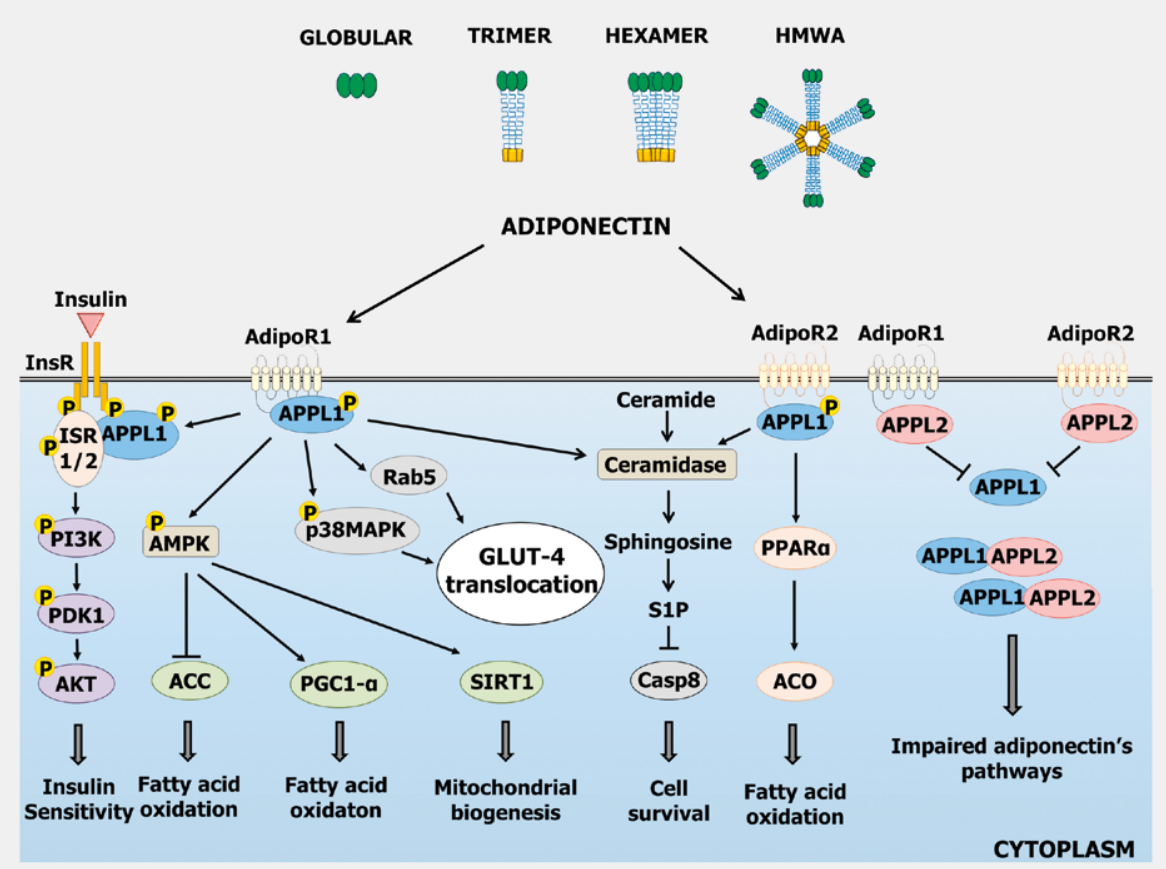

- Fig. 1 Adiponectin pathways: Adiponectins are secreted into the bloodstream in different forms: globular, trimer, hexamer, and high-molecular-weight (HMW) multimer. Adiponectin's biological effects are mediated by its specific receptors: AdipoR1, AdipoR2 and T-cadherin (not shown). AdipoR1/R2 recruits the adaptor protein APPL1, which initiates AMPK activation. APPL1 also activates other signaling molecules, such as p38 MAPK, PPAR $\alpha$ and the RAS-associated protein Rab5. These pathways induce fatty-acid oxidation and glucose uptake, by GLUT-4 translocation to the cell membrane. In addition, adiponectin's pathway enhances mitochondrial biogenesis by sirtuin 1 activation. Adiponectin exerts anti-apoptotic actions by stimulation of ceramidase activity and the formation of sphingosine-1-phosphate (S1P). It was shown that adiponectin effectively induces insulin sensitivity by interaction with ISR 1/2. On the other hand, APPL2 binds to AdipoR1 and AdipoR2 blocking adiponectin signaling by both competitive inhibition of APPL1 and formation of APPL1/APPL2 heterodimers, thereby reducing APPL1 availability to AdipoRs. InsR: Insulin receptor; ISR 1/2: Insulin receptor substrate 1 and 2; APPL 1/2: Adaptor protein, phosphotyrosine interacting with PH domain and leucine zipper 1 and 2; PI3K: Phosphoinositide 3-kinase; PDK1: 3-Phosphoinositide-dependent protein kinase-1; AKT or PKB: Protein kinase B; AMPK: AMP-activated protein kinase; p38MAPK: p38 Mitogen-activated protein kinase; ACC: Acetylcarboxylase; PGC1- $\alpha$, Peroxisome proliferator-activated receptor gamma coactivator 1-alpha; SIRT1: Sirtuin 1; Casp8: Caspase 8; GLUT-4: Glucose transporter type 4; PPAR- $\alpha$ : Peroxisome proliferator-activated receptor alpha; ACO: Enzyme acyl-CoA oxidase.

ing to insulin resistance and marked glucose intolerance [41]. Furthermore, insulin-resistant obese mice show lower AdipoR1 protein levels in skeletal muscle and reduced adiponectin sensitivity [40]. Mice with muscle-specific insulin resistance show high circulating adiponectin levels and adiponectin resistance. The PI3K/Akt/ FOXO1 pathway mediates the insulin-inhibited expression of AdipoR1 in skeletal muscle [42]. In humans with genetically defective insulin receptors, high plasma adiponectin levels and low leptin concentrations were reported [43].

Taken together, high insulin levels related to insulin resistance downregulate AdipoR1 expression and adiponectin pathway, consequently reduces peroxisome proliferator-activated receptor coactivator-1 $\alpha$ (PGC1- $\alpha$ ), with a subsequent increase in ceramides, ultimately leading to adiponectin resistance ( Fig. 3) [44]. Despite the experimental evidence suggesting that increased insulin concentrations down-regulate AdipoR1 and AdipoR2, the question of whether adiponectin resistance precedes and is associated with the development of insulin resistance remains. In this regard, Mullen and colleagues [27] conducted a time-course high-fat diet (HFD) trial in rats for 3 days, 2 weeks, or 4 weeks, to determine the onset of adiponectin resistance and to identify the resulting temporal changes in lipid metabolism and insulin sensitivity in skeletal muscle. Adiponectin resistance emerged very quickly (as early as 3 days in HFD animals), clearly demonstrating that it precedes the accumulation of skeletal muscle lipids and insulin resistance in high-fatfed rats.

\section{Adiponectin resistance and chronic heart failure - An example of a biologically two-faced molecule}

Recently, adiponectin received the eponym "Janus molecule" [45], referring to its dual performance in the natural history of certain chronic diseases, for example in chronic heart failure (CHF). At an early stage of $\mathrm{CHF}$, when the ventricular ejection fraction is preserved (HFpEF), circulating adiponectin levels are lower and adiponectin exerts a cardioprotective effect. In contrast, hyperadiponectinemia, reflecting adiponectin resistance, and a possible detrimental effect of adiponectin on the myocardium characterize advanced stages of heart failure reduced ejection fraction (HFrEF) $[45,46]$. It has also been shown that the biological response of Ad- 


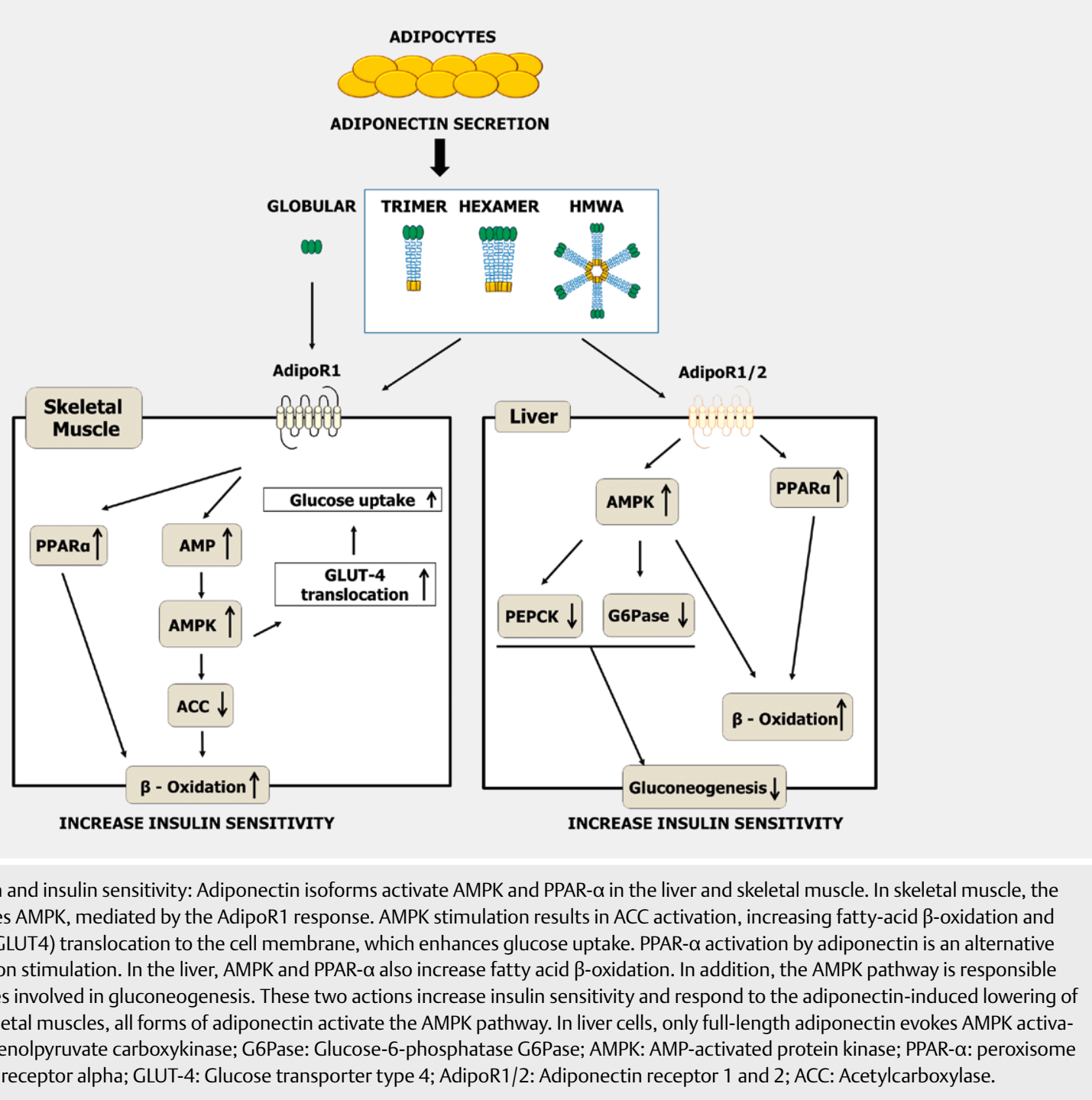

ipoRs to adiponectin in skeletal muscle tissue obtained from patients with advanced heart failure is significantly impaired [45].

Adiponectin levels vary not only with the functional capacity of the heart, but also with the patient's metabolic status. Adiponectin levels are increased in patients with advanced heart failure, but to a lesser extent in diabetics than in non-diabetics [47]. Experimental protocols have demonstrated that higher adiponectin levels are associated with severe myocardial dysfunction, advanced heart failure, and higher mortality rates. Obese diabetic mice induced by a high-fat diet, when submitted to myocardial infarction reperfusion (MIR) injury, needed a three-fold higher dose of adiponectin to achieve significant cardioprotection, compared with mice on a normal diet treated with a standard adiponectin dose. In addition, the obese diabetic mice showed a significant reduction in both the AMPK-dependent and AMPK-independently mediated cardioprotective effects of adiponectin [48]. These findings strongly suggest that adiponectin resistance develops in the obese diabetic heart.
Van Berendoncks and co-workers [49] reported a local adiponectin system in skeletal-muscle biopsies obtained from patients with HFrEF, which also showed high circulating levels of adiponectin. A five-fold increase in the expression of adiponectin mRNA and protein were observed in these patients, although the mRNA and protein expression levels of skeletal muscle AdipoR1 were decreased. The authors also reported a deactivated PPAR $\alpha / A M P K$ pathway and down-regulation of several enzymes involved in the metabolism of free fatty acids (FFA) and glucose, thus characterizing a skeletal muscle metabolic deficiency and a functional adiponectin resistance state. On the other hand, insulin resistance increases in parallel with the severity of heart failure and accelerates the development of skeletal-muscle wasting in cardiac patients [50].

Contributing to better comprehension of adiponectin resistance in the evolution of heart failure, Cui and colleagues [51] have shown, in hyperinsulinemia rat models induced by chronic infusion of insulin, increased serum and myocardial adiponectin concentrations and decreases in skeletal muscle and myocardial AdipoR1 ex- 

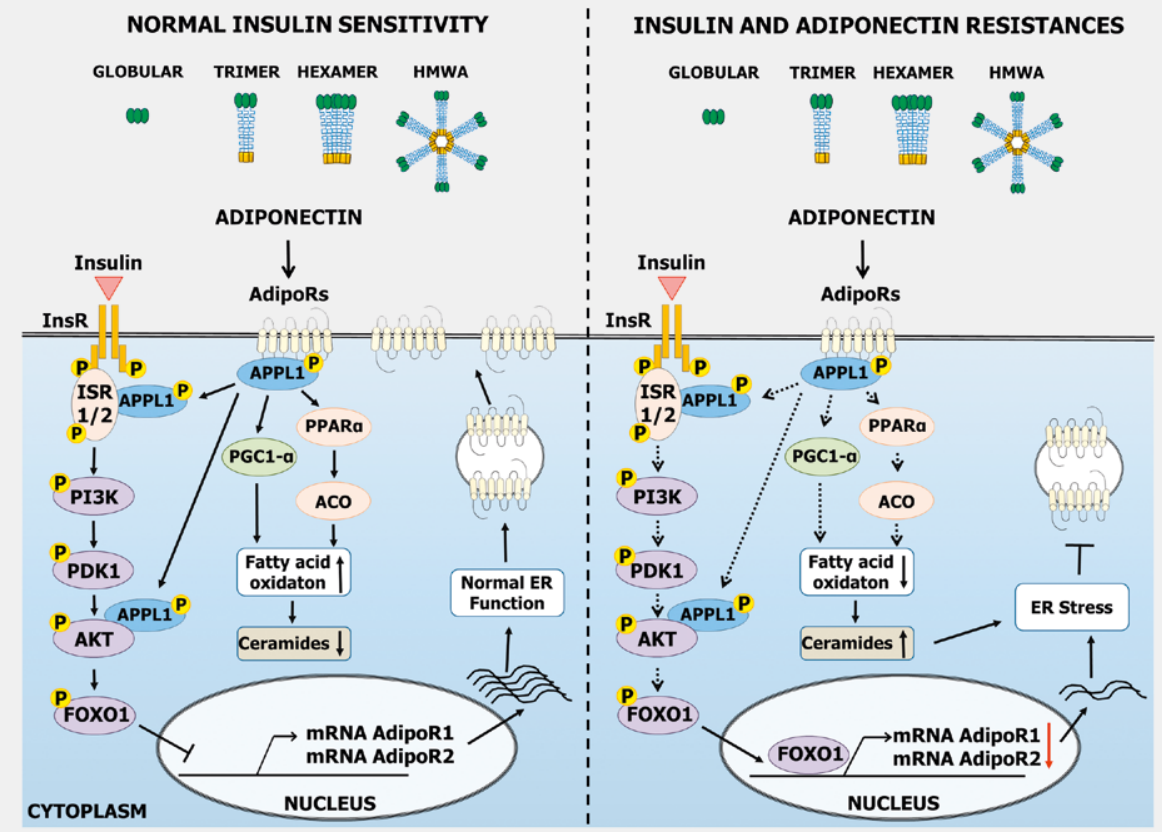

- Fig. 3 Mechanisms of insulin and adiponectin resistance: In normal insulin sensitivity, adiponectin enhances insulin sensitivity by interacting with both ISRs and AKT. On the other hand, insulin activation of PI3K/Akt pathway results in FOXO1 phosphorylation and recruitment to cytosol. In normal conditions, this upregulates AdipoRs expression enhancing adiponectin's pathway (arrows) that controls fatty acid oxidation, decreasing cells ceramides internal levels. Under insulin and adiponectin resistant state, insulin pathway is less active (dotted arrows) decreasing FOXO1 phosphorylation, inducing its nucleartranslocation. In this case, FOXO1 reduces the expression of AdipoR1 and AdipoR2 with subsequent attenuation of adiponectin signaling (dotted arrows). AdipoRs downregulation attenuates PGC1- $\alpha$ and PPAR $\alpha$ activity, with a subsequent increase in ceramides levels which in turns leads to endoplasmic reticulum stress and a major reduction in adiponectin's sensitivity. AdipoRs: Adiponectin's receptors; InsR: Insulin receptor; ISR 1/2: Insulin receptor substrate 1 and 2; APPL1: Adaptor protein, phosphotyrosine interacting with PH domain and leucine zipper 1; PI3K: Phosphoinositide 3-kinase; PDK1: 3-Phosphoinositide-dependent protein kinase-1; AKT or PKB: Protein kinase B; PGC1- $\alpha$ : Peroxisome proliferator-activated receptor gamma coactivator 1- $\alpha$; PPAR- $\alpha$ : peroxisome proliferator-activated receptor alpha; ACO, enzyme acyl-CoA oxidase.

pression and AMP-activated protein kinase phosphorylation. These results indicate that despite an increase in serum and myocardial adiponectin, the decreased AdipoR1 expression and AMPK phosphorylation suggest an adiponectin-resistance state occurring in the early stage of hyperinsulinemia.

However, the association of hyperadiponectinemia and heart failure must be interpreted with caution. For example, it is not yet clear whether high adiponectin levels are deleterious or beneficial to heart failure patients, especially in those exhibiting reduced ejection fractions. On the other hand, no relationship of adiponectin levels with incident heart failure was found in data from the Framingham Offspring Study [52]. In addition, a U-shaped association between adiponectin and total mortality has been reported in older men and women [53]. Similarly, results from the Physicians' Health Study have shown data consistent with a J-shaped association between total adiponectin and the risk of heart failure among male physicians in the USA [54]. In a similar context, a nonlinear relationship between total and high-molecular-weight adiponectin with incident T2DM, considered a major risk factor for heart failure, was reported in older adults [55]. Taken together, this apparently contradictory effect of adiponectin may be related to the evolution and metabolic conditions of the natural history of heart failure, reinforcing a nonlinear relationship of adiponectin and heart failure.
Some evidence suggests that adiponectin resistance can be reversed to adiponectin sensitivity. In patients with advanced heart failure, the favorable impact of hemodynamic correction (mechanical unloading) through implantation of a ventricular assistance device (VAD) may reduce tissue inflammation and adiponectin resistance in these patients. In 2012, Khan and colleagues [56] demonstrated, after implantation of a VAD in a failing myocardium, a significant reduction in adiponectin levels, reversed down-regulation of AdiopoR1 and AdipoR2, besides decreasing adiponectin expression in adipose tissue, and decreased insulin resistance. Notably, macrophage infiltration in adipose tissue was higher in heart-failure patients compared with control subjects, but was normalized after VAD implantation. On the other hand, in patients with $\mathrm{HFrEF}$, endurance and resistance-training exercise reduces circulating adiponectin concentrations, and normalized the muscle-specific expression of adiponectin, AdipoR1, and genes involved in lipid and glucose metabolism [57].

Recently, Waragai and collaborators [58] suggest that hyperadiponectinemia might also be associated with Alzheimer disease (AD). This group has proposed that adiponectin might be involved during the reproductive stage promoting the amyloidogenic evolvability, which may subsequently manifest as AD in senescence by the antagonistic pleiotropy mechanism. Although adiponectin has 
been considered a neuroprotective cytokine in experimental protocols, the result of a large prospective study conducted by the Mayo Clinic Study of Aging ( $n=535$, aged 70 years, without dementia) showed that higher levels of adiponectin were associated with significant neuroimaging changes for hippocampal and cortical volumes, and cognitive deficits [59]. In addition, there are evidence suggesting that $\mathrm{AD}$ and $\mathrm{CHF}$ overlap and converge from the pathophysiological point of view [60]. It has been speculated that adiponectin paradox in certain chronic diseases, such as CHF and chronic kidney disease, might be attributed to the stimulation of the amyloidogenic evolvability [58].

\section{Adiponectin Resistance and Adiponectin Paradox}

Early findings from in vitro studies support the cardioprotective effects of adiponectin [61,62]. Experimental and clinical evidence has also shown that low adiponectin levels predict the risk of cardiovascular events [63] and myocardium infarction [64], and are inversely associated with early-onset coronary artery disease (CAD) and multiple coronary-artery stenosis [65].

However, as already mentioned, in late stages of certain chronic diseases, high adiponectin levels are associated with low functional capacity of organs, as occurs in patients with chronic heart failure [66]. Moreover, hyperadiponectinemia is associated with a poor prognosis in elderly subjects with weight loss, skeletal-muscle wasting, and disability [45], and in patients with cachexia in the terminal phase of cancer [67]. Lee and co-workers [68] have shown in a population recruited from the Hong Kong Diabetes Registry the adiponectin paradox associated with the development of new cases of cancer and cancer related deaths in type 2 diabetic patients. They concluded that hyperadiponectinemia could be a risk biomarker of incident cancer in type 2 diabetes.

These results have been confirmed by numerous prospective studies and meta-analyses, now focusing on the association of adiponectin levels with primary outcomes (all-cause mortality and cardiovascular mortality rates), scientifically more robust than surrogate endpoints. In this context, the apparently contradictory impact of a reverse positive, rather than expected negative, relationship between adiponectin and mortality rate came to be designated as the "adiponectin paradox" $[25,45,69]$.

The causes of the adiponectin paradox are still under debate, but some hypotheses have been formulated. Tsukamoto and colleagues [70] have shown that atrial natriuretic peptide (NAP) and brain natriuretic peptide (BNP) enhance the expression and secretion of adiponectin in vitro by human adipocytes, and in subjects with CHF. In the same report, the infusion of recombinant adiponectin increased the adiponectin serum concentration and had a beneficial therapeutic effect on cardiomyocytes of patients with heart failure [70]. Hyperadiponectinemia might also be a compensatory mechanism in insulin-resistance states, reflecting a lowered activity of insulin/IGF-1 receptor signaling pathways, which are essential for homeostatic modulation of the central nervous system and cardiovascular systems [71].

As stated above, besides adipose tissue, adiponectin can be expressed and secreted ectopically in tissues such as bone marrow, osteoblasts, and vascular and skeletal muscle cells. A recent study showed that bone-marrow adipose tissue (MAT) is a major source of serum HMW adiponectin in subjects with anorexia nervosa [72]. MAT is also increased in aging- associated leanness, which may explain the higher level of adiponectin in frail elderly persons [73]. Last, plasma adiponectin might be up-regulated because of adiponectin resistance, which reflects a decreased expression and/or activity of adiponectin-receptor signaling, increased endoplasmic reticulum stress, and inflammation.

Biologically, it can be speculated whether in individuals at greater risk of death, increased adiponectin levels would not represent an in extremis failing attempt to overcome adiponectin resistance in adipose tissue, vasculature, heart, kidney and skeletal muscle [69]. Indeed, this response would be beneficial and expected, as it could lead to prolongation of life. Consistent with this hypothesis, adiponectin has been considered a protective molecule for disease prevention in aging, particularly in healthy centenarians [74]. In humans, adiponectin/AdipoR signaling activates the AMPK-SIRT1 pathway and positively regulates the PPAR pathway, reducing oxidative stress. These signaling pathways have been considered essential for longevity [75]. Unfortunately, none of these data allow robust conclusions. So, further efforts are needed to unravel the role of adiponectin in cardiometabolic health and, most importantly, its paradoxical association with increased mortality risk.

\section{Association of Circulating Adiponectin with Different Clinical Outcomes - Expected and Unexpected Results}

\section{Cross-sectional and prospective studies}

Earlier studies conducted in a population highly prone to developing obesity and exhibiting the highest reported prevalence of T2DM in the world, the Pima Indians of Arizona, showed that Pima adults had lower plasma adiponectin levels compared with Caucasians [76]. Hypoadiponectinemia in these patients predicted a reduction in insulin sensitivity [77]. Subsequently, the inverse relationship between plasma adiponectin and adiposity and fasting-insulin levels recorded in adults was confirmed in Pima children [78]. These findings agreed with previous observational studies in Japan $[79,80]$, and were later confirmed in several studies, including in multi-ethnic participants from the Dallas Heart Study [81].

A close correlation of hypoadiponectinemia with a decrease in insulin sensitivity and T2DM has been described in various population-based studies [82]. A meta-analysis emphasized the inverse association between total plasma adiponectin levels and the incidence of T2DM among various populations [83]. Conversely, a high level of adiponectin predicted a reduced risk for T2DM and metabolic syndrome $[84,85]$.

Another recent meta-analysis analyzed the accuracy and validity of circulating adiponectin in the diagnosis of metabolic syndrome, and showed that plasma levels of this adipokine may be a potentially useful biomarker for the detection of individuals with metabolic syndrome, especially in populations with significant insulin resistance. In addition, the risk of metabolic syndrome associated with hypoadiponectinemia was higher in men than in women [86]. In addition, results from our group have shown that the HMWA/HOMA-IR ratio, when compared with HOMA-IR (a robust 
clinical and epidemiological marker of metabolic syndrome) and the active circulating form of adiponectin (HMWA), was a sensitive predictor for metabolic syndrome, being the only marker that was significantly associated with all the individual components of metabolic syndrome [87].

\section{Results of Mendelian randomization studies}

Although some epidemiological evidence from observational and longitudinal studies shows an association of hypoadiponectinemia with insulin resistance, metabolic syndrome, and T2DM, these results have the limitations of classical observational epidemiology. Overall, they point to the role of adiponectin as a predictive biomarker, but do not support a causal relationship. Fortunately, determining the causal relationship between an intermediate phenotype and the outcome of interest became possible after genetic variants were identified that consistently affect the outcome of certain traits. Thus, using genes as instruments for inferring causal relationships in epidemiological studies, through Mendelian randomization [88], has been considered a valid approach to explore the relationships between adiponectin and metabolic outcomes.

In this respect, studies of candidate genes and GWA have identified common and rare variants at the ADIPOQ locus that are associated with the serum adiponectin level [89]. Additional contributing loci have been identified by large-scale GWA meta-analyses [90-92]. In the same context, Dastani and colleagues [92], using datasets from an international consortium, found strong evidence of an association between several single-nucleotide polymorphism (SNP) adiponectin-decreasing gene scores and hypertriglyceridemia, low-HDL-cholesterol, higher HOMA-IR, increased risk of T2DM and increased waist-to-hip ratio, all component variables of metabolic syndrome. Although the Mendelian randomization approach has limitations, it provides consistent evidence to accept or reject a causal relationship between intermediary biomarkers and complex diseases [93].

One of the first studies to explore the relationship of adiponectin to metabolic-outcome phenotypes using Mendelian randomization was conducted in a population comprised of Canadians of European, South Asian, Chinese, and Aboriginal origins who had previously participated in two cross-sectional studies of cardiovascular disease. The results showed an association of the functional promoter SNP rs266729 in the ADIPOQ gene with lower serum adiponectin and increased insulin resistance as evaluated by $\mathrm{HO}$ MA-IR, thus suggesting a causal relationship between adiponectin level and insulin resistance [93]. Gao and co-workers confirmed these results, demonstrating in a Caucasian population of Swedish men a strong causal relationship of genetic variants of the ADIPOQ gene with adiponectin levels and insulin sensitivity [94].

However, entirely different results were obtained from a larger multicenter population sample from different countries, which indicated no causal relationship between a genetic-risk score using available common SNPs associated with adiponectin levels and insulin resistance (as measured by fasting insulin), and T2DM, thus providing no consistent evidence that genetically influenced decreased circulating adiponectin levels increase the risk of insulin resistance or diabetes [95]. More recently, the metabolic profiling of adiponectin levels was analyzed in a large population of European adults who have participated in six longitudinal studies and one
GWA study. Using multivariate regression analyses, higher circulating adiponectin levels were associated with a salutary metabolic profile. However, these findings have not been confirmed by Mendelian randomization using genetic variants in the vicinity of the adiponectin-encoding gene. These results indicate that adiponectin levels are not a key determinant of metabolic profile, but rather are an epiphenomenon in the context of cardiometabolic metabolic traits [96].

Although pioneering studies have pointed toward the predictive value of adiponectin levels in assessing the risk of myocardial infarction [97] and the extent of coronary heart disease (CHD) [98], it is still controversial whether adiponectin is causally related to CHD. Borges and colleagues conducted a Mendelian randomization study using data from the GWA studies consortia CARDIoGRAM and CARDIoGRAMplusC4DMetabochip, with more than 250000 cases (CHD) and control subjects, the majority of European ancestry. There was no evidence that genetic predisposition to increased adiponectin levels reduced the risk of CHD, when the analysis was restricted to ADIPOQ SNP. When the analysis used variants associated with adiponectin across the genome, a protective effect of adiponectin appeared, which disappeared after adjustment for CHD predictors [99].

The Mendelian randomization approach can be considered as nature's counterpart to randomized controlled studies, and has been widely used to investigate the possible causal relationship between intermediate phenotypes with metabolic and cardiovascular outcomes [100]. In addition, Mendelian randomization studies are not as vulnerable as observational studies, and can improve causal inference [101]. In sum, Mendelian randomization is a means through which genetic epidemiology may contribute to a better understanding of the environmental determinants of disease [102].

\section{Circulating adiponectin levels and risk of death - arguments favoring the adiponectin paradox}

Presently, consistent lines of evidence demonstrate that hyperadiponectinemia is an important independent positive risk predictor for all-cause mortality and cardiovascular mortality in patients with advanced heart failure, particularly when associated with wasting skeletal muscle [69].

The association of adiponectin with fatal outcomes was initially described by Efstathiou and coworkers, who reported that low adiponectin plasma levels were independently associated with an increased risk of mortality after a first ischemic stroke [103]. Subsequently, however, the majority of studies have shown a positive association or no association of adiponectin with all-cause mortality and cardiovascular mortality [69]. These associations were described in a wide range of clinical conditions, in different genders and diverse ethnicities, and in the general population of older individuals $[20,53,104-106]$; older patients with weight loss, low skeletal-muscle mass, and poor physical functioning [24]; elderly people with T2DM [107]; T2DM patients with acute coronary syndrome [21]; elderly men with chronic heart failure [104]; patients with chronic heart failure and cachexia [108]; myocardial infarction patients [109]; stable ischemic heart disease [110]; individuals with diabetes and recent acute coronary syndrome [21]; acute stroke patients [111]; peripheral artery diseases [112]; Japanese hemodialysis patients [113]; end-stage renal disease in patients with T1DM 
and T2DM [114]; cancer [115]; women with breast cancer [116]; and patients with colorectal cancer [117].

Most of these studies indicate that adiponectin levels, regardless of whether they were measured as total, HMW, or LMW adiponectin, are valuable predictive biomarkers for all-cause mortality and cardiovascular mortality. In the analysis of these results, however, one must take into account the presence of confounding factors, for example the frequent use of polypharmacy, particularly in populations of frail elderly, diabetics and cardiovascular patients. BMI is another confounding factor, with some studies demonstrating an association of adiponectin with mortality rate in specific BMI subgroups [105] and others not [21,110].

Recent evidence has confirmed the causal relationship between adiponectin and cardiovascular mortality. The Gargano Heart Study, employing the Mendelian randomization approach, has shown a positive causal-effect relationship between serum adiponectin and cardiovascular mortality in subjects with type 2 diabetes. Regression-modeling studies have shown that BMI, HbA1c, total cholesterol, HDL-cholesterol, triglycerides, insulin therapy and hypertension, and rs 822354 polymorphism in the ADIPOQ locus, as well as the genetic equivalent of total adiponectin change, were significantly associated with cardiovascular mortality [118]. These results, based on the methodological robustness of genetic variants affecting the outcomes, point to a deleterious action of adiponectin on metabolic and cardiovascular pathophysiological processes. A systematic review and meta-analysis conducted by Scarale and collaborators demonstrated a significant increase of $24 \%$ and $28 \%$ in pooled hazard ratios for all-cause and cardiovascular mortality, respectively [23]. Interestingly, in a subgroup analysis, the all-cause mortality rate was significantly reduced after adjusting for natriuretic peptides (NPs). This raises the possibility that the association of adiponectin with mortality may be modulated by NPs.

In fact, Scarale's meta-analysis extended and confirmed previous meta-analyses published in 2013 [119], although both were based on less evidence, considering the number of collected prospective studies.

\section{Perspectives}

Given the evidence that loss of AdipoRs functions might be related to insulin-resistant states, it has been postulated that agonists of these receptors could be a promising alternative for the treatment of dysmetabolic conditions, such as metabolic syndrome and T2DM. Recently, AdipoRon, an orally active synthetic peptide AdipoRs agonist [120], has emerged as a possible candidate for treatment and prevention of insulin-resistance states, cancer, depression and Alzheimer's disease, all conditions that may be associated with adiponectin resistance [74].

The extensive literature on AdipoRon is presently limited to in vitro and in vivo animal models. AdipoRon mimics the beneficial metabolic effects of adiponectin, activating AMPK, PPAR- $\alpha$, and the transcriptional co-activator PGC-1, which enhance mitochondrial proliferation and energy metabolism [121]. Okada-Iwabu and colleagues have reported that mice fed a high-fat diet and treated with AdipoRon showed a significant improvement of skeletal muscle and liver-mediated glucose metabolism, thereby ameliorating insulin resistance and glucose intolerance, besides increasing fatty acid $\beta$-oxidation [120]. AdipoRon has also shown a renoprotective role against lipotoxicity and oxidative stress in mice with diabetic nephropathy, by ameliorating glomerular endothelial cells and podocyte injury through activation of the intracellular $\mathrm{Ca}^{2+} /$ LKB1-AMPK/PPARa pathway [48].

Other favorable results of AdipoRon, not restricted to the metabolic area, were reported in experimental conditions such as pancreatic cancer cells [122], depression [123], liver injury, liver inflammation [124], and cutaneous-thickness fibrosis in a murine model of systemic sclerosis [125]. Notwithstanding these favorable results, they do not provide consistent evidence that interventions aimed at increasing adiponectin levels will improve the metabolic profile in humans.

As mentioned above, findings from Mendelian randomization studies do not support a salutary role of adiponectin in the incidence of T2DM [95] or a protective effect in CHD [96]. Thus, the pharmaceutical industry's efforts to prioritize the development of drugs that increase adiponectin levels seem, at this point, premature. Coincidentally, a search in the archives of the US National Library of Medicine through October 2019 found no references to clinical trials conducted with AdipoRon (US National Library of Medicine 2020, available online at: https://clinicaltrials.gov - accessed February 21, 2020).

Adiponectin gene therapy has been also successfully used to ameliorate obesity induced insulin resistance and T2DM, in animal models [126, 127]. Recently, the development of targeted gene modification via chimeric genome editing tools enabled investigators to use engineered animals to better understand the pathophysiological process of a variety of diseases. The clustered regularly interspaced short palindromic repeat (CRISPR)-associated 9 (Cas9) nuclease and the transcription activator-like effector nucleases (TALEN) platforms have been successfully applied for animal models to better understand human metabolic diseases such as obesity, insulin and adiponectin resistances and T2DM $[128,129]$. They also represent an extremely promising future for the development of novel and effective therapies in clinical practice.

Adiponectin antagonist has emerged as an alternative therapeutic approach to conditions that evolve into adiponectin resistance. Based on previous evidence showing that circulating adiponectin concentrations are elevated in advanced stages of disease in several clinical settings, it has been proposed that adiponectin-receptor antagonists might play an important role in these terminal conditions. Compared with the literature on adiponectin agonists, there are a limited number of reports on adiponectin receptor antagonists [130]. Even considering their potential indication in specific situations, this proposal would be restricted to diseases characterized by adiponectin overproduction, as in cases of severe rheumatoid arthritis [131], or when overexpression of AdipoRs occurs, as in cartilage osteoarthritis [132].

\section{Conclusions}

For years, adiponectin was considered a healthy adipocytokine because of its anti-inflammatory properties, favorable effects on intermediary metabolism, and cardioprotection. Several observational and prospective studies have shown that low levels of circulating adiponectin were associated with insulin resistance, 
Healthy subjects in terms of functional capacity of organs and metabolic status Initial stages of organ and metabolic dysfunctions

Plasma levels of adiponectin are usually low.*

Hypoadiponectinemia has been reported in

$\checkmark$ Reduced insulin sensitivity states, glucose intolerance and early stages of non-complicated T2DM

$\checkmark$ Metabolic syndrome

$\checkmark$ Hypertension

$\checkmark$ Visceral obesity

$\checkmark$ NAFLD

$\checkmark$ CHD and non-complicated MI

$\checkmark$ HFpEF
Late stages of organ dysfunction and metabolic disorders

Plasma levels of adiponectin are high.*

Hyperadiponectinemia has been reported in

$\checkmark$ Insulin and adiponectin resistant states

$\checkmark$ Humans with defective insulin receptor

$\checkmark$ CHD, MI and CHF with reduced ejection fraction

$\checkmark$ End-stage renal disease

$\checkmark$ Stroke

$\checkmark$ Weight loss and skeletal muscle wasting

specially in the elderly

$\checkmark$ Terminal cancer

$\checkmark$ Prospective studies associated with primary outcomes (all-cause mortality and cardiovascular mortality rates)

$\checkmark$ Tumor suppressive

- Fig. 4 The natural history of circulating levels of adiponectin according to the stages of functional capacity of organs and systems: Adiponectin has been considered a salutary adipocytokine a. Results from experimental protocols and early observational and prospective studies have shown that low levels of adiponectin are associated with weight excess, insulin resistant, glucose intolerance, T2DM, dyslipidemia, and cardiovascular events, usually with preserved heart function (non-fatal CHD and MI) b. In contrast, hyperadiponectinemia has been frequently associated with late stages chronic heart failure (usually with reduced ejection fraction), cardiovascular mortality, end stage renal disease, skeletal muscle mass wasting of elderly, cachexia of terminal stages of cancer, and all-cause mortality (c). Recent findings from Mendelian randomization studies have not supported a salutary role of adiponectin in the incidence of T2DM or a protective effect in CHD. * The cut-off point considered as low or high adiponectin depends on each class of study-specific distribution. T2DM: Type 2 diabetes mellitus; CHD: Coronary heart disease; HFpEF: Heart failure with ventricular ejection fraction preserved; CHF: Chronic heart failure; MI: Myocardial infarction; NAFLD: Non-alcoholic fatty liver disease.

hypertension, metabolic syndrome, and T2DM and its cardiovascular complications.

Due to the nature of these studies, a causal relationship between adiponectin with systemic metabolic profile and cardiovascular outcomes has not been effectively proven. Findings from Mendelian randomization studies, a robust genetic approach to test causality, have not supported a salutary role of adiponectin in the incidence of T2DM or a protective effect in CHD. Adiponectin was therefore considered as a mere biomarker of components of metabolic syndrome, including insulin resistance, hypertension and dyslipidemia, or an epiphenomenon in the context of cardiometabolic metabolic traits.

Paradoxically and counterintuitively, in late stages of chronic diseases, high adiponectin levels are associated with low functional capacity of organs, as in patients with chronic heart failure, in the elderly exhibiting weight loss and skeletal-muscle wasting, and in cachectic subjects in the terminal phase of cancer. These results were confirmed by several prospective studies and meta-analyses, now focusing on the association of adiponectin levels with primary outcomes (all-cause mortality and cardiovascular mortality rates), which is scientifically more consistent than surrogate endpoints. In a broader context, this contradictory behavior of adiponectin has been termed the adiponectin paradox ( $\mathbf{F i g . ~ 4 ) . ~ I n ~}$ terms of biological plausibility, there is an extensive literature indicating that the adiponectin paradox is closely related to insulin resistance and adiponectin resistance. Although important advances have been achieved in the last three decades, thanks to basic scientific research and clinical studies, there still remain a number of open questions about adiponectin. We expect that the ongoing dialogue among basic scientists, clinical researchers, and epidemiologists can consistently further elucidate the concept of adiponectin paradox.

\section{Author Contributions}

All authors contributed equally to the manuscript. The illustrations were designed by Rômulo Sperduto Dezonne.

\section{Funding Information}

This work was supported by the following Brazilian agencies: 1. Coordenação de Aperfeiçoamento de Pessoal de Nível Superior (CAPES); 2. Fundação de Amparo à Pesquisa do Estado do Rio de Janeiro (FAPERJ).

\section{Acknowledgements}

We would like to thank Mauricio de Assis Fontes for his helpful assistance in the preparation of this manuscript. 


\section{Conflict of Interest}

The authors declare that they have no conflict of interest.

\section{References}

[1] $\mathrm{Ng} \mathrm{M}$, Fleming T, Robinson M et al. Global, regional, and nationa prevalence of overweight and obesity in children and adults during 1980-2013: A systematic analysis for the Global Burden of Disease Study 2013. Lancet 2014; 384: 766-781

[2] WHO. World Health Organization: Obesity and overweight. https:// www.who.int/news-room/fact-sheets/detail/obesity-and-overweight; Accessed in: July 30, 2020

[3] Apovian CM, Okemah J, O'Neil PM. Body weight considerations in the management of type 2 diabetes. Adv Ther 2019; 36: 44-58

[4] McMurray J], Petrie MC, Murdoch DR et al. Clinical epidemiology of heart failure: Public and private health burden. Eur Heart ] 1998; 19 (Suppl P): P9-P16

[5] Wang YC, McPherson K, Marsh T et al. Health and economic burden of the projected obesity trends in the USA and the UK. Lancet 2011; 378: 815-825

[6] Lau DC, Dhillon B, Yan H et al. Adipokines: Molecular links between obesity and atheroslcerosis. Am J Physiol Heart Circ Physiol 2005; 288: $\mathrm{H} 2031-\mathrm{H} 2041$

[7] Li ], Xue YM, Zhu B et al. Rosiglitazone elicits an adiponectin-mediated insulin-sensitizing action at the adipose tissue-liver axis in otsuka long-evans tokushima fatty rats. J Diabetes Res 2018; 4627842

[8] Iannitti T, Graham A, Dolan S. Adiponectin-mediated analgesia and anti-inflammatory effects in rat. PLoS One 2015; 10: e0136819

[9] Nomura-Nakayama K, Adachi H, Miyatake $\mathrm{N}$ et al. High molecular weight adiponectin inhibits vascular calcification in renal allograft recipients. PLoS One 2018; 13: e0195066

[10] Potenza MA, Sgarra L, Nacci C et al. Activation of AMPK/SIRT1 axis is required for adiponectin-mediated preconditioning on myocardial ischemia-reperfusion (I/R) injury in rats. PLoS One 2019; 14: e0210654

[11] Shibata R, Ouchi N, Murohara T. Adiponectin and cardiovascular disease. Circ J 2009; 73: 608-614

[12] Bruun JM, Lihn AS, Verdich C et al. Regulation of adiponectin by adipose tissue-derived cytokines: In vivo and in vitro investigations in humans. Am J Physiol Endocrinol Metab 2003; 285: E527-E533

[13] Turer AT, Scherer PE. Adiponectin: Mechanistic insights and clinical implications. Diabetologia 2012; 55: 2319-2326

[14] Nishida M, Funahashi T, Shimomura I. Pathophysiological significance of adiponectin. Med Mol Morphol 2007; 40: 55-67

[15] Engl ], Sturm W, Sandhofer A et al. Effect of pronounced weight loss on visceral fat, liver steatosis and adiponectin isoforms. Eur J Clin Invest 2008; 38: 238-244

[16] Ryu HK, Yu SY, Park JS et al. Hypoadiponectinemia is strongly associated with metabolic syndrome in Korean type 2 diabetes patients. J Am Coll Nutr 2010; 29: 171-178

[17] Patel DA, Srinivasan SR, Xu JH et al. Adiponectin and its correlates of cardiovascular risk in young adults: The Bogalusa Heart Study. Metabolism 2006; 55: 1551-1557

[18] Francischetti EA, Celoria BM, Duarte SF et al. Hypoadiponectinemia is associated with blood pressure increase in obese insulin-resistant individuals. Metabolism 2007; 56: 1464-1469

[19] Kyrou I, Tsantarlioti O, Panagiotakos DB et al. Adiponectin circulating levels and 10-year (2002-2012) cardiovascular disease incidence: The ATTICA Study. Endocrine 2017; 58: 542-552
[20] Kizer JR, Benkeser D, Arnold AM et al. Associations of total and high-molecular-weight adiponectin with all-cause and cardiovascular mortality in older persons: The Cardiovascular Health Study. Circulation 2012; 126: 2951-2961

[21] Bergmark BA, Cannon CP, White WB et al. Baseline adiponectin concentration and clinical outcomes among patients with diabetes and recent acute coronary syndrome in the EXAMINE trial. Diabetes Obes Metab 2017; 19: 962-969

[22] Uetani E, Tabara Y, Kawamoto R et al. CDH13 genotype-dependent association of high-molecular weight adiponectin with all-cause mortality: The J-SHIPP study. Diabetes Care 2014; 37: 396-401

[23] Scarale MG, Fontana A, Trischitta V et al. Circulating adiponectin levels are paradoxically associated with mortality rate. A systematic review and meta-analysis. J Clin Endocrinol Metab 2019; 104: 1357-1368

[24] Baker JF, Newman AB, Kanaya A et al. The adiponectin paradox in the elderly: Associations with body composition, physical functioning, and mortality. J Gerontol A Biol Sci Med Sci 2019; 74: 247-253

[25] Sattar N, Nelson SM. Adiponectin, diabetes, and coronary heart disease in older persons: Unraveling the paradox. J Clin Endocrinol Metab 2008; 93: 3299-3301

[26] Engin A. Adiponectin-Resistance in Obesity. Adv Exp Med Biol 2017; 960: 415-441

[27] Mullen KL, Pritchard J, Ritchie I et al. Adiponectin resistance precedes the accumulation of skeletal muscle lipids and insulin resistance in high-fat-fed rats. Am J Physiol Regul Integr Comp Physiol 2009; 296: R243-R251

[28] Yamaguchi N, Argueta JG, Masuhiro Y et al. Adiponectin inhibits Toll-like receptor family-induced signaling. FEBS Lett 2005; 579: 6821-6826

[29] Ohashi K, Parker JL, Ouchi N et al. Adiponectin promotes macrophage polarization toward an anti-inflammatory phenotype. J Biol Chem 2010; 285: 6153-6160

[30] Wong WT, Tian XY, Xu A et al. Adiponectin is required for PPARgamma-mediated improvement of endothelial function in diabetic mice. Cell Metab 2011; 14: 104-115

[31] Ohashi K, Shibata R, Murohara T et al. Role of anti-inflammatory adipokines in obesity-related diseases. Trends Endocrinol Metab 2014; 25: 348-355

[32] Esmaili S, Xu A, George J. The multifaceted and controversial immunometabolic actions of adiponectin. Trends Endocrinol Metab 2014; 25: 444-451

[33] Park PH, McMullen MR, Huang $\mathrm{H}$ et al. Short-term treatment of RAW264.7 macrophages with adiponectin increases tumor necrosis factor-alpha (TNF-alpha) expression via ERK1/2 activation and Egr-1 expression: Role of TNF-alpha in adiponectin-stimulated interleukin-10 production. J Biol Chem 2007; 282: 21695-21703

[34] Yamamoto K, Kiyohara T, Murayama Y et al. Production of adiponectin, an anti-inflammatory protein, in mesenteric adipose tissue in Crohn's disease. Gut 2005; 54: 789-796

[35] Liu D, Luo S, Li Z. Multifaceted roles of adiponectin in rheumatoid arthritis. Int Immunopharmacol 2015; 28: 1084-1090

[36] Rao M, Li L, Tighiouart $\mathrm{H}$ et al. Plasma adiponectin levels and clinical outcomes among haemodialysis patients. Nephrol Dial Transplant 2008; 23: 2619-2628

[37] Kaser S, Moschen A, Kaser A et al. Circulating adiponectin reflects severity of liver disease but not insulin sensitivity in liver cirrhosis. J Intern Med 2005; 258: 274-280

[38] Waschki B, Kirsten A, Holz O et al. Physical activity is the strongest predictor of all-cause mortality in patients with COPD: A prospective cohort study. Chest 2011; 140: 331-342 
[39] Putti R, Migliaccio V, Sica R et al. Skeletal muscle mitochondrial bioenergetics and morphology in high fat diet induced obesity and insulin resistance: Focus on dietary fat source. Front Physiol 2015; 6: 426

[40] Tsuchida A, Yamauchi T, Ito Y et al. Insulin/Foxo1 pathway regulates expression levels of adiponectin receptors and adiponectin sensitivity. J Biol Chem 2004; 279: 30817-30822

[41] Yamauchi T, Nio Y, Maki T et al. Targeted disruption of AdipoR1 and AdipoR2 causes abrogation of adiponectin binding and metabolic actions. Nat Med 2007; 13: 332-339

[42] Kim CH, Pennisi P, Zhao H et al. MKR mice are resistant to the metabolic actions of both insulin and adiponectin: Discordance between insulin resistance and adiponectin responsiveness. Am J Physiol Endocrinol Metab 2006; 291: E298-E305

[43] Semple RK, Soos MA, Luan J et al. Elevated plasma adiponectin in humans with genetically defective insulin receptors. J Clin Endocrinol Metab 2006; 91: 3219-3223

[44] Chen J, Ma X, Yang Y et al. Glycine enhances expression of adiponectin and IL-10 in 3T3-L1 adipocytes without affecting adipogenesis and lipolysis. Amino Acids 2018; 50: 629-640

[45] Sente T, Van Berendoncks AM, Hoymans VY et al. Adiponectin resistance in skeletal muscle: Pathophysiological implications in chronic heart failure. J Cachexia Sarcopenia Muscle 2016; 7: 261-274

[46] Nakamura T, Funayama $\mathrm{H}$, Kubo $\mathrm{N}$ et al. Association of hyperadiponectinemia with severity of ventricular dysfunction in congestive heart failure. Circ J 2006; 70: 1557-1562

[47] Baldasseroni S, Antenore A, Di Serio C et al. Adiponectin, diabetes and ischemic heart failure: a challenging relationship. Cardiovasc Diabetol 2012; 11: 151

[48] Kim Y, Lim JH, Kim MY et al. The adiponectin receptor agonist adiporon ameliorates diabetic nephropathy in a model of type 2 diabetes. J Am Soc Nephrol 2018; 29: 1108-1127

[49] Van Berendoncks AM, Garnier A, Beckers P et al. Functional adiponectin resistance at the level of the skeletal muscle in mild to moderate chronic heart failure. Circ Heart Fail 2010; 3: 185-194

[50] Wang X, Hu Z, Hu J et al. Insulin resistance accelerates muscle protein degradation: Activation of the ubiquitin-proteasome pathway by defects in muscle cell signaling. Endocrinology 2006; 147: 41604168

[51] Cui XB, Wang C, Li L et al. Insulin decreases myocardial adiponectin receptor 1 expression via $\mathrm{PI} 3 \mathrm{~K} / \mathrm{Akt}$ and FoxO1 pathway. Cardiovasc Res 2012; 93: 69-78

[52] Frankel DS, Vasan RS, D’Agostino RB Sr. et al. Resistin, adiponectin, and risk of heart failure the Framingham offspring study. J Am Coll Cardiol 2009; 53: 754-762

[53] Kizer JR, Arnold AM, Jenny NS et al. Longitudinal changes in adiponectin and inflammatory markers and relation to survival in the oldest old: The Cardiovascular Health Study All Stars study. J Gerontol A Biol Sci Med Sci 2011; 66: 1100-1107

[54] Djousse L, Wilk JB, Hanson NQ et al. Association between adiponectin and heart failure risk in the physicians' health study. Obesity (Silver Spring) 2013; 21: 831-834

[55] Kizer JR, Arnold AM, Benkeser D et al. Total and high-molecular-weight adiponectin and risk of incident diabetes in older people. Diabetes Care 2012; 35: 415-423

[56] Khan RS, Kato TS, Chokshi A et al. Adipose tissue inflammation and adiponectin resistance in patients with advanced heart failure: Correction after ventricular assist device implantation. Circ Heart Fail 2012; 5: 340-348

[57] Van Berendoncks AM, Beckers P, Hoymans VY et al. Exercise training reduces circulating adiponectin levels in patients with chronic heart failure. Clin Sci (Lond) 2010; 118: 281-289
[58] Waragai M, Ho G, Takamatsu Y et al. Adiponectin paradox in Alzheimer's disease; relevance to amyloidogenic evolvability? Front Endocrinol (Lausanne) 2020; 11: 108

[59] Wennberg AM, Gustafson D, Hagen CE et al. Serum adiponectin levels, neuroimaging, and cognition in the Mayo Clinic Study of Aging. J Alzheimers Dis 2016; 53: 573-581

[60] Franciosa JA. Epidemiologic patterns, clinical evaluation, and long-term prognosis in chronic congestive heart failure. Am J Med 1986; 80: 14-21

[61] Ouchi N, Shibata R, Walsh K. Cardioprotection by adiponectin. Trends Cardiovasc Med 2006; 16: 141-146

[62] Shibata R, Sato K, Pimentel DR et al. Adiponectin protects against myocardial ischemia-reperfusion injury through AMPK- and COX-2-dependent mechanisms. Nat Med 2005; 11: 1096-1103

[63] Chen CY, Asakura M, Asanuma $\mathrm{H}$ et al. Plasma adiponectin levels predict cardiovascular events in the observational Arita Cohort Study in Japan: The importance of the plasma adiponectin levels. Hypertens Res 2012; 35: 843-848

[64] Hatano Y, Matsumoto M, Ishikawa S et al. Plasma adiponectin level and myocardial infarction: The JMS Cohort Study. J Epidemiol 2009; 19: 49-55

[65] Lindberg S, Pedersen SH, Mogelvang R et al. Usefulness of adiponectin as a predictor of all cause mortality in patients with ST-segment elevation myocardial infarction treated with primary percutaneous coronary intervention. Am J Cardiol 2012; 109: 492-496

[66] Laoutaris ID, Vasiliadis IK, Dritsas A et al. High plasma adiponectin is related to low functional capacity in patients with chronic heart failure. Int J Cardiol 2010; 144: 230-231

[67] Ye J, Liang Z, Liang Q et al. Adiponectin is associated with poor prognosis in carcinoma patients: Evidence from a meta-analysis. Lipids Health Dis 2015; 14: 154

[68] Lee CH, Lui DTW, Cheung CYY et al. Higher circulating adiponectin concentrations predict incident cancer in type 2 diabetes - The adiponectin paradox. J Clin Endocrinol Metab 2020; 105. doi:10.1210/clinem/dgaa075

[69] Menzaghi C, Trischitta V. The adiponectin paradox for all-cause and cardiovascular mortality. Diabetes 2018; 67: 12-22

[70] Tsukamoto O, Fujita M, Kato M et al. Natriuretic peptides enhance the production of adiponectin in human adipocytes and in patients with chronic heart failure. J Am Coll Cardiol 2009; 53: 2070-2077

[71] Waragai M, Ho G, Takamatsu Y et al. Importance of adiponectin activity in the pathogenesis of Alzheimer's disease. Ann Clin Transl Neurol 2017; 4: 591-600

[72] Cawthorn WP, Scheller EL, Learman BS et al. Bone marrow adipose tissue is an endocrine organ that contributes to increased circulating adiponectin during caloric restriction. Cell Metab 2014; 20: 368-375

[73] Tanaka Y, Kita S, Nishizawa $\mathrm{H}$ et al. Adiponectin promotes muscle regeneration through binding to T-cadherin. Sci Rep 2019; 9: 16

[74] Waragai M, Ho G, Takamatsu Y et al. Dual-therapy strategy for modification of adiponectin receptor signaling in aging-associated chronic diseases. Drug Discov Today 2018; 23: 1305-1311

[75] Iwabu M, Okada-Iwabu M, Yamauchi T et al. Adiponectin/adiponectin receptor in disease and aging. NPJ Aging Mech Dis 2015; 1: 15013

[76] Weyer C, Funahashi T, Tanaka S et al. Hypoadiponectinemia in obesity and type 2 diabetes: close association with insulin resistance and hyperinsulinemia. J Clin Endocrinol Metab 2001; 86: 1930-1935

[77] Stefan N, Vozarova B, Funahashi T et al. Plasma adiponectin concentration is associated with skeletal muscle insulin receptor tyrosine phosphorylation, and low plasma concentration precedes a decrease in whole-body insulin sensitivity in humans. Diabetes 2002; 51: $1884-1888$ 
[78] Stefan N, Bunt JC, Salbe AD et al. Plasma adiponectin concentrations in children: Relationships with obesity and insulinemia. J Clin Endocrinol Metab 2002; 87: 4652-4656

[79] Arita Y, Kihara S, Ouchi N et al. Paradoxical decrease of an adipose-specific protein, adiponectin, in obesity. Biochem Biophys Res Commun 1999; 257: 79-83

[80] Hotta K, Funahashi T, Arita Y et al. Plasma concentrations of a novel, adipose-specific protein, adiponectin, in type 2 diabetic patients. Arterioscler Thromb Vasc Biol 2000; 20: 1595-1599

[81] Turer AT, Khera A, Ayers CR et al. Adipose tissue mass and location affect circulating adiponectin levels. Diabetologia 2011; 54 : 2515-2524

[82] Zhu W, Cheng KK, Vanhoutte PM et al. Vascular effects of adiponectin: Molecular mechanisms and potential therapeutic intervention. Clin Sci (Lond) 2008; 114: 361-374

[83] Li S, Shin HJ, Ding EL et al. Adiponectin levels and risk of type 2 diabetes: A systematic review and meta-analysis. JAMA 2009; 302: 179-188

[84] Knobler H, Benderly M, Boyko V et al. Adiponectin and the development of diabetes in patients with coronary artery disease and impaired fasting glucose. Eur J Endocrinol 2006; 154: 87-92

[85] Wang Y, Meng RW, Kunutsor SK et al. Plasma adiponectin levels and type 2 diabetes risk: A nested case-control study in a Chinese population and an updated meta-analysis. Sci Rep 2018; 8: 406

[86] Liu Z, Liang S, Que S et al. Meta-analysis of adiponectin as a biomarker for the detection of metabolic syndrome. Front Physiol 2018; 9: 1238

[87] de Abreu VG, Martins CJM, de Oliveira PAC et al. High-molecular weight adiponectin/HOMA-IR ratio as a biomarker of metabolic syndrome in urban multiethnic Brazilian subjects. PLoS One 2017; 12: e0180947

[88] Lawlor DA, Harbord RM, Sterne JA et al. Mendelian randomization: Using genes as instruments for making causal inferences in epidemiology. Stat Med 2008; 27: 1133-1163

[89] Heid IM, Henneman P, Hicks A et al. Clear detection of ADIPOQ locus as the major gene for plasma adiponectin: Results of genome-wide association analyses including 4659 European individuals. Atherosclerosis 2010; 208: 412-420

[90] Richards JB, Waterworth D, O’Rahilly S et al. A genome-wide association study reveals variants in ARL15 that influence adiponectin levels. PLoS Genet 2009; 5: e1000768

[91] Wu Y, Li Y, Lange EM et al. Genome-wide association study for adiponectin levels in Filipino women identifies $\mathrm{CDH} 13$ and a novel uncommon haplotype at KNG1-ADIPOQ. Hum Mol Genet 2010; 19: 4955-4964

[92] Dastani Z, Hivert MF, Timpson N et al. Novel loci for adiponectin levels and their influence on type 2 diabetes and metabolic traits: A multi-ethnic meta-analysis of 45,891 individuals. PLoS Genet 2012; 8: e1002607

[93] Mente A, Meyre D, Lanktree MB et al. Causal relationship between adiponectin and metabolic traits: A Mendelian randomization study in a multiethnic population. PLoS One 2013; 8: e66808

[94] Gao H, Fall T, van Dam RM et al. Evidence of a causal relationship between adiponectin levels and insulin sensitivity: A Mendelian randomization study. Diabetes 2013; 62: 1338-1344

[95] Yaghootkar H, Lamina C, Scott RA et al. Mendelian randomization studies do not support a causal role for reduced circulating adiponectin levels in insulin resistance and type 2 diabetes. Diabetes 2013; 62: 3589-3598

[96] Borges MC, Barros AJD, Ferreira DLS et al. Metabolic profiling of adiponectin levels in adults: Mendelian randomization analysis. Circ Cardiovasc Genet 2017; 10: e001837. doi:10.1161/circgenetics.117.001837
[97] Pischon T, Girman C], Hotamisligil GS et al. Plasma adiponectin levels and risk of myocardial infarction in men. JAMA 2004; 291: 17301737

[98] von Eynatten M, Schneider JG, Humpert PM et al. Serum adiponectin levels are an independent predictor of the extent of coronary artery disease in men. J Am Coll Cardiol 2006; 47: 2124-2126

[99] Borges MC, Lawlor DA, de Oliveira C et al. Role of adiponectin in coronary heart disease risk: A Mendelian Randomization Study. Circ Res. 2016; 119: 491-499. doi:10.1161/CIRCRESAHA116.308716

[100] Wensley F, Gao P, Burgess $S$ et al. Association between $C$ reactive protein and coronary heart disease: Mendelian randomisation analysis based on individual participant data. BMJ 2011; 342: d548

[101] Smith GD, Ebrahim S. 'Mendelian randomization’: Can genetic epidemiology contribute to understanding environmental determinants of disease? Int J Epidemiol 2003; 32: 1-22

[102] Mucci LA, Wedren S, Tamimi RM et al. The role of gene-environment interaction in the aetiology of human cancer: Examples from cancers of the large bowel, lung and breast. J Intern Med 2001; 249: 477-493

[103] Efstathiou SP, Tsioulos DI, Tsiakou AG et al. Plasma adiponectin levels and five-year survival after first-ever ischemic stroke. Stroke 2005; 36: 1915-1919

[104] Wannamethee SG, Whincup PH, Lennon L et al. Circulating adiponectin levels and mortality in elderly men with and without cardiovascular disease and heart failure. Arch Intern Med 2007; 167: 1510-1517

[105] Choi SH, Ku EJ, Hong ES et al. High serum adiponectin concentration and low body mass index are significantly associated with increased all-cause and cardiovascular mortality in an elderly cohort, "adiponectin paradox": The Korean Longitudinal Study on Health and Aging (KLoSHA). Int J Cardiol 2015; 183: 91-97

[106] Rizza S, Cardellini M, Farcomeni A et al. Low molecular weight adiponectin increases the mortality risk in very old patients. Aging Dis 2018; 9: 946-951

[107] Singer JR, Palmas W, Teresi J et al. Adiponectin and all-cause mortality in elderly people with type 2 diabetes. Diabetes Care 2012; 35: $1858-1863$

[108] McEntegart MB, Awede B, Petrie MC et al. Increase in serum adiponectin concentration in patients with heart failure and cachexia: relationship with leptin, other cytokines, and B-type natriuretic peptide. Eur Heart J 2007; 28: 829-835

[109] Cavusoglu E, Ruwende C, Chopra V et al. Adiponectin is an independent predictor of all-cause mortality, cardiac mortality, and myocardial infarction in patients presenting with chest pain. Eur Heart J 2006; 27: 2300-2309

[110] Beatty AL, Zhang MH, Ku IA et al. Adiponectin is associated with increased mortality and heart failure in patients with stable ischemic heart disease: data from the Heart and Soul Study. Atherosclerosis 2012; 220: 587-592

[111] Nagasawa H, Yokota C, Toyoda K et al. High level of plasma adiponectin in acute stroke patients is associated with stroke mortality. J Neurol Sci 2011; 304: 102-106

[112] Dieplinger B, Haltmayer M, Poelz W et al. Value of adiponectin as predictor of 5-year all-cause mortality in patients with symptomatic peripheral arterial disease: Results from the Linz Peripheral Arterial Disease (LIPAD) study. Clin Chim Acta 2009; 408: 87-91

[113] Matsumoto H, Kimura T, Nishida S et al. Experimental evidence characterizing pressure fluctuations at the seafloor-water interface induced by an earthquake. Sci Rep 2018; 8: 16406

[114] Jorsal A, Tarnow L, Frystyk J et al. Serum adiponectin predicts all-cause mortality and end stage renal disease in patients with type I diabetes and diabetic nephropathy. Kidney Int 2008; 74: 649-654 
[115] Kojima R, Ukawa S, Zhao W et al. Association of Adiponectin With Cancer and All-Cause Mortality in a Japanese Community-Dwelling Elderly Cohort: A Case-Cohort Study. J Epidemiol 2018; 28: 367-372

[116] Duggan C, Irwin ML, Xiao L et al. Associations of insulin resistance and adiponectin with mortality in women with breast cancer. J Clin Oncol 2011; 29: 32-39

[117] Chong DQ, Mehta RS, Song M et al. Prediagnostic plasma adiponectin and survival among patients with colorectal cancer. Cancer Prev Res (Phila) 2015; 8: 1138-1145

[118] Ortega Moreno L, Copetti M, Fontana A et al. Evidence of a causal relationship between high serum adiponectin levels and increased cardiovascular mortality rate in patients with type 2 diabetes. Cardiovasc Diabetol 2016; 15: 17

[119] Sook Lee E, Park SS, Kim E et al. Association between adiponectin levels and coronary heart disease and mortality: a systematic review and meta-analysis. Int J Epidemiol 2013; 42: 1029-1039

[120] Okada-Iwabu M, Yamauchi T, Iwabu M et al. A small-molecule AdipoR agonist for type 2 diabetes and short life in obesity. Nature 2013; 503: 493-499

[121] Okada-Iwabu M, Iwabu M, Ueki K et al. Perspective of small-molecule AdipoR agonist for type 2 diabetes and short life in obesity. Diabetes Metab J 2015; 39: 363-372

[122] Akimoto M, Maruyama R, Kawabata Y et al. Antidiabetic adiponectin receptor agonist AdipoRon suppresses tumour growth of pancreatic cancer by inducing RIPK1/ERK-dependent necroptosis. Cell Death Dis 2018; 9: 804

[123] Nicolas S, Debayle D, Bechade C et al. Adiporon, an adiponectin receptor agonist acts as an antidepressant and metabolic regulator in a mouse model of depression. Transl Psychiatry 2018; 8: 159
[124] Wang Y, Wan Y, Ye G et al. Hepatoprotective effects of AdipoRon against d-galactosamine-induced liver injury in mice. Eur J Pharm Sci 2016; 93: 123-131

[125] Yamashita T, Lakota K, Taniguchi T et al. An orally-active adiponectin receptor agonist mitigates cutaneous fibrosis, inflammation and microvascular pathology in a murine model of systemic sclerosis. Sci Rep 2018; 8: 11843

[126] Kandasamy AD, Sung MM, Boisvenue J] et al. Adiponectin gene therapy ameliorates high-fat, high-sucrose diet-induced metabolic perturbations in mice. Nutr Diabetes 2012; 2: e45

[127] Banerjee A, Sharma D, Trivedi R et al. Treatment of insulin resistance in obesity-associated type 2 diabetes mellitus through adiponectin gene therapy. Int J Pharm 2020; 583: 119357

[128] Zheng Q, Lin J, Huang J et al. Reconstitution of UCP1 using CRISPR/ Cas9 in the white adipose tissue of pigs decreases fat deposition and improves thermogenic capacity. Proc Natl Acad Sci U S A 2017; 114 : E9474-E9482

[129] Li H, Yang Y, Hong W et al. Applications of genome editing technology in the targeted therapy of human diseases: mechanisms, advances and prospects. Signal Transduct Target Ther 2020; 5: 1

[130] Otvos L Jr. Potential Adiponectin Receptor Response Modifier Therapeutics. Front Endocrinol (Lausanne) 2019; 10: 539

[131] Ebina K, Fukuhara A, Ando W et al. Serum adiponectin concentrations correlate with severity of rheumatoid arthritis evaluated by extent of joint destruction. Clin Rheumatol 2009; 28: 445-451

[132] Kang EH, Lee Y], Kim TK et al. Adiponectin is a potential catabolic mediator in osteoarthritis cartilage. Arthritis Res Ther 2010; 12: R231 